\title{
Describing the Characteristics and Scope of Nursing Practice in the Psychiatric Department of Special Functioning Hospitals in Japan - Research Protocol
}

Akiko Nosaki 1), Kanae Moriuchi 2)3), Izumi Kohara4)5)

1) Graduate School of Nursing, Chiba University, Japan

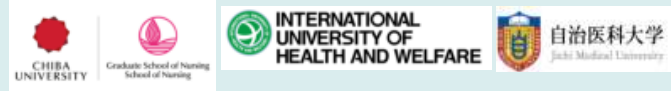

2) Department of Nursing, Chiba University Hospital, Japan

3) Graduate School of Health and Welfare Sciences, International University of Health and Welfare, Japan

4) School of Nursing, Jichi Medical University

5) Support Center for Clinical Investigation, Jichi Medical University

\section{Objectives:}

> Background: The Japanese government has classified the hospitals to systematize the functioning of medical facilities, and the Minister of Health, Labour and Welfare has started approving hospitals that are capable of providing medical care, developing advanced medical technologies, and conducting advanced medical care training as Special Functioning Hospitals (SFHs) since 1993 (Table. 1). For a leading hospital, requirements for being approved as an SFH are having 400 or more beds, and twice as many doctors as those in ordinary hospitals, and more regulations concerning the facilities (Table. 2). In 2018, the total number of psychiatric beds in those $75 \mathrm{SFHs}$ ' psychiatric department was 3,308(Table. 3)(Nosaki \& Moriuchi, 2018). Not only the medication but also the high level and advanced psychiatric nursing care are required there. However, most nurses who work there are generalists.

$>$ Aim of this study: To describe and identify and the characteristics and the scope of nursing practice at the department of psychiatry among SFHs in Japan to optimize clinical practice, including nursing care there.

\section{Methods:}

This study used a descriptive design. All nurse managers among the 75 SFHs' psychiatric department were eligible. Along with the original self-administered questionnaire, demographic data; clinical experience of the nurse managers; number of beds, nurses, and psychiatrists; average duration of hospitalization; nurse-to-patient ratio; and type of targeted psychiatric disease and treatment, difficulties in nursing care will be collected as data(Table. 4).

Table. 3 Clinical indicators of psychiatric department of SFHs Clinical indicators of Psychiatric department of SFHs

Number of prefecture

Number of SFH per prefecture

Type of organizer

national university corporation

municipal university corporation

incorporated educational institution

Minister of Defense

national research and development agency

Mean number of total beds

Mean number of psychiatric beds

Mean number of psychiatrists(including part-time)

Mean number of psychiatric beds per psychiatrist $(\mathrm{N}=75)$

Table. 1 Types of medical institutions in Japan

\begin{tabular}{|l|}
\hline Medical Institutions in Japan \\
\hline Hospitals (20 or more beds) \\
\hline General hospitals \\
\hline $\begin{array}{c}\text { Special functioning hospitals } \\
\text { (providing advanced medical treatments) }\end{array}$ \\
$\begin{array}{c}\text { Regional medical care support hospitals } \\
\text { (supporting family doctors and family dentists who are taking roles of local medical treatments) }\end{array}$ \\
\hline $\begin{array}{l}\text { Psychiatric hospitals } \\
\text { (hospitals with psychiatric wards only) }\end{array}$ \\
\hline $\begin{array}{c}\text { Tuberculosis hospitals } \\
\text { (hospitals with tuberculosis only) }\end{array}$ \\
\hline Clinics (0 to 19 beds) \\
\hline Clinics with beds ( 1 to 19 beds) \\
\hline Clinics without beds ( 0 beds) \\
\hline
\end{tabular}

(the Ministry of Health, Labour, and Welfare in Japan) https://www.mh/w.go.jp/english/wp/wp-hw2/part2/p3_0006.pdf

Table. 2 Definition of the Special Functioning Hospitals

\section{Detailed Information of Special Functioning Hospitals}

Purpose

As part of efforts to systematize medical facility functions, the Minister of Health, Labour and Welfare approves individual having capabilities of providing advanced medical care, development of advanced technologies, and conducting advanced medical care training.

\section{Roles}

$>$ Provide advanced medical care

Develop/evaluate advanced medical technologies

$>$ Conduct advanced medical care training

\section{Requirements for Approval}

> Having capabilities of providing, developing, evaluating, and conduct training of advanced medical care

> Providing medical care to patients who are referred to by other hospitals and clinics

> Number of beds: Must have 400 or more beds

$>$ Staff deployment: Number of Doctors is about twice of ordinary hospitals, etc.

- Facilities: Must have intensive care units, sterile rooms, and drug information management rooms

$>$ etc.

Results:

The results of this research will contribute to developing an educational program for promoting psychiatric nursing practice at SFHs.

Table. 4 Methods of the study

\section{Methods of the study}

Design: descriptive study

Sampling: complete study

Subjects: nurse manager among the 75 SFHs' psychiatric department Data collection:

$>$ tool: original self-administered questionnaire

$>$ data items:
- about nurse manager: demographic data, clinical experience

- about the psychiatric department: clinical statistics (average duration of hospitalization etc.), number and profession of medical staffs, nurse-to-patient ratio, targeted psychiatric disease, types of treatment,

- about the nursing care: staff nurses' clinical experience, difficulties in nursing care

- about the educational needs of nurses: educational needs for psychiatric nursing care at SFH 